\title{
Diffuse Pancreatic Cancer Mimicking Autoimmune Pancreatitis
}

\author{
Hideaki Miyoshi, Masataka Kano, Sanshiro Kobayashi, Takashi Ito, Masataka Masuda, \\ Toshiyuki Mitsuyama, Shinji Nakayama, Tsukasa Ikeura, Masaaki Shimatani, \\ Kazushige Uchida, Makoto Takaoka and Kazuichi Okazaki
}

\begin{abstract}
:
The characteristic finding of sausage-shaped pancreas or capsule-like rim facilitates the diagnosis of autoimmune pancreatitis. We herein report a case of a 67-year-old man showing a sausage-shaped, enlarged pancreas with a capsule-like rim on computed tomography. Furthermore, endoscopic retrograde cholangiopancreatography demonstrated diffuse narrowing of the main pancreatic duct, in addition to stenosis of the lower bile duct. Finally, we were able to diagnose pancreatic cancer in this patient by an endoscopic ultrasoundguided fine-needle aspiration biopsy following peroral cholangioscopy and bile cytology. This report emphasizes the significance of pathological confirmation before starting treatment, even in cases with diffuse pancreatic enlargement.
\end{abstract}

Key words: diffuse pancreatic cancer, autoimmune pancreatitis, sausage-shaped pancreas, capsule-like rim, EUS-FNAB, POCS

(Intern Med 58: 2523-2527, 2019)

(DOI: 10.2169/internalmedicine.2689-19)

\section{Introduction}

Autoimmune pancreatitis (AIP) is a specific type of pancreatitis that is characterized by focal or diffuse pancreatic enlargement, irregular narrowing of the main pancreatic duct, elevated serum immunoglobulin G4 (IgG4), other organ involvement conditions (e.g. sclerosing cholangitis), and a dramatic response to steroid therapy. Depending on its morphology, AIP is categorized into diffuse-type and focal/ segmental-type.

Focal/segmental-type AIP can share overlapping clinical features with pancreatic cancer (PC), which is an aggressive tumor with a 5 -year survival rate of $<10 \%$. It has been reported that between $2 \%$ and $5 \%$ of patients undergoing resection for suspected PC were diagnosed with focal/ segmental-type AIP after surgery $(1,2)$. The accurate diagnosis of pancreatic enlargement using various imaging modalities can therefore avoid unnecessary surgical resection or delays in cancer treatment due to diagnostic steroid trials.
For diffuse-type AIP, a sausage-shaped pancreas with a capsule-like rim on computed tomography (CT) is a highly specific and typical feature. Consequently, it is rarely necessary to differentiate diffuse-type AIP from PC.

We herein report a rare case of PC presenting with manifestations similar to diffuse-type AIP that was successfully diagnosed using an endoscopic ultrasound-guided fineneedle aspiration biopsy (EUS-FNAB) to assess the pancreatic enlargement and peroral cholangioscopy (POCS) and bile cytology to demonstrate cancer invasion in the lower bile duct.

\section{Case Report}

A 67-year-old man presented with icterus and upper abdominal pain and was referred to our hospital for further investigation of obstructive jaundice and pancreatic enlargement on abdominal ultrasonography (US). He had a history of hypertension and urolithiasis. The man's family history was unremarkable. The patient was a smoker (10 cigarettes 


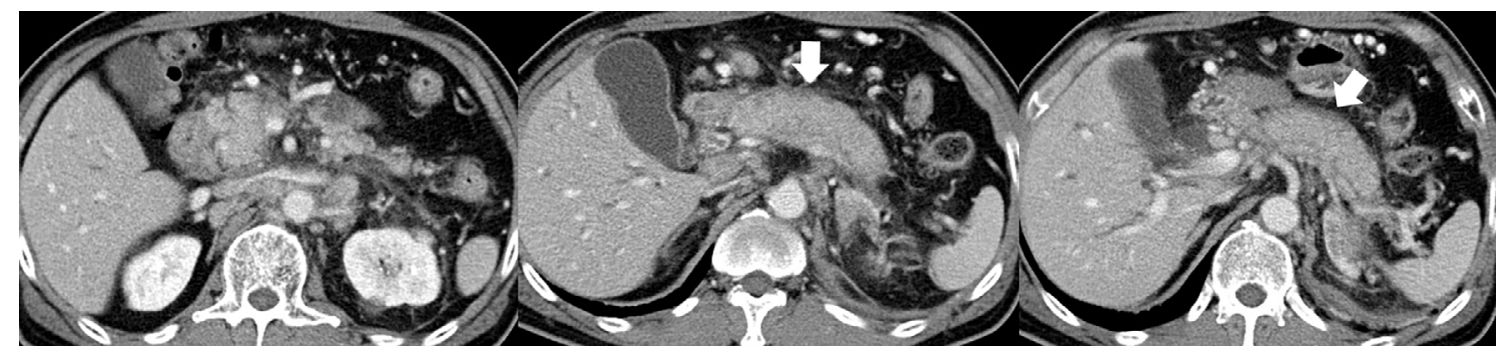

Figure 1. Contrast-enhanced CT scans showing delayed enhancement of a sausage-shaped, enlarged pancreas with a capsule-like rim at the surface (arrows) and dilation of the intrahepatic bile duct.
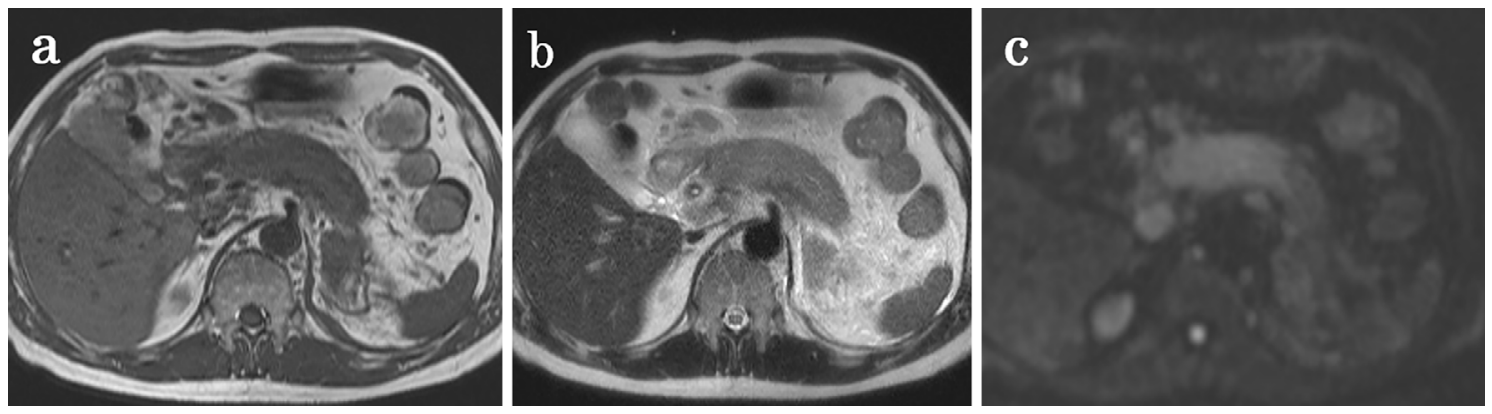

Figure 2. MRI scans showing a diffusely enlarged pancreas with low intensity on T1-weighted imaging (a), slightly high intensity on T2-weighted imaging (b), and high intensity on DWI (c).
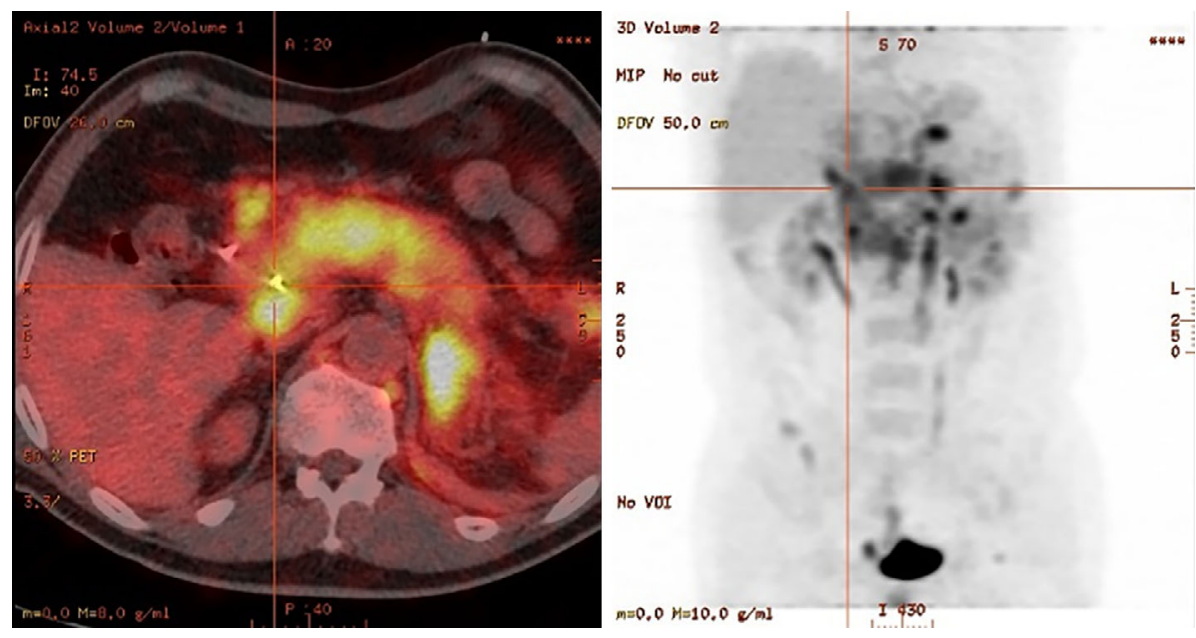

Figure 3. PET-CT scans showing a diffuse pattern of FDG uptake in the pancreas and multiple foci of FDG uptake in the lymph nodes.

a day) and denied regular alcohol consumption.

On an examination, he had clinical icterus and upper abdominal tenderness. Laboratory test results showed bilirubinemia $(9.6 \mathrm{mg} / \mathrm{dL}$, reference values $<1.2 \mathrm{mg} / \mathrm{dL})$, elevated serum levels of hepatobiliary enzymes, elevated HbA1c (7.2\%, reference range 4.6-6.2\%), and elevated values of the tumor markers carcinoembryonic antigen (CEA) (28.8 ng/ $\mathrm{mL}$, reference values $<5 \mathrm{ng} / \mathrm{mL})$ and CA19-9 $(76.6 \mathrm{U} / \mathrm{mL}$, reference values $<37 \mathrm{U} / \mathrm{mL}$ ). Serum IgG4 and amylase levels were within the reference ranges. Enhanced CT showed a delayed enhanced sausage-shaped, enlarged pancreas including a capsule-like rim at the surface and a dilated intra- hepatic bile duct (Fig. 1). Magnetic resonance imaging (MRI) showed a diffusely enlarged pancreas with a low intensity on T1-weighted imaging (Fig. 2a), slightly high intensity on T2-weighted imaging (Fig. 2b), and high intensity on diffusion-weighted imaging (DWI) (Fig. 2c). The pancreatic duct was not visualized on magnetic resonance cholangiopancreatography (data not shown). ${ }^{18}$ F-fluorodeoxyglucose positron emission tomography (FDG-PET) showed a diffuse FDG uptake in the pancreas, with a maximum standardized uptake value $\left(\mathrm{SUV}_{\max }\right)$ of 8.6, and multiple foci of FDG uptake in the lymph nodes, with an $\mathrm{SUV}_{\max }$ of 10.0 (Fig. 3). Endoscopic retrograde cholangiopancreatography 


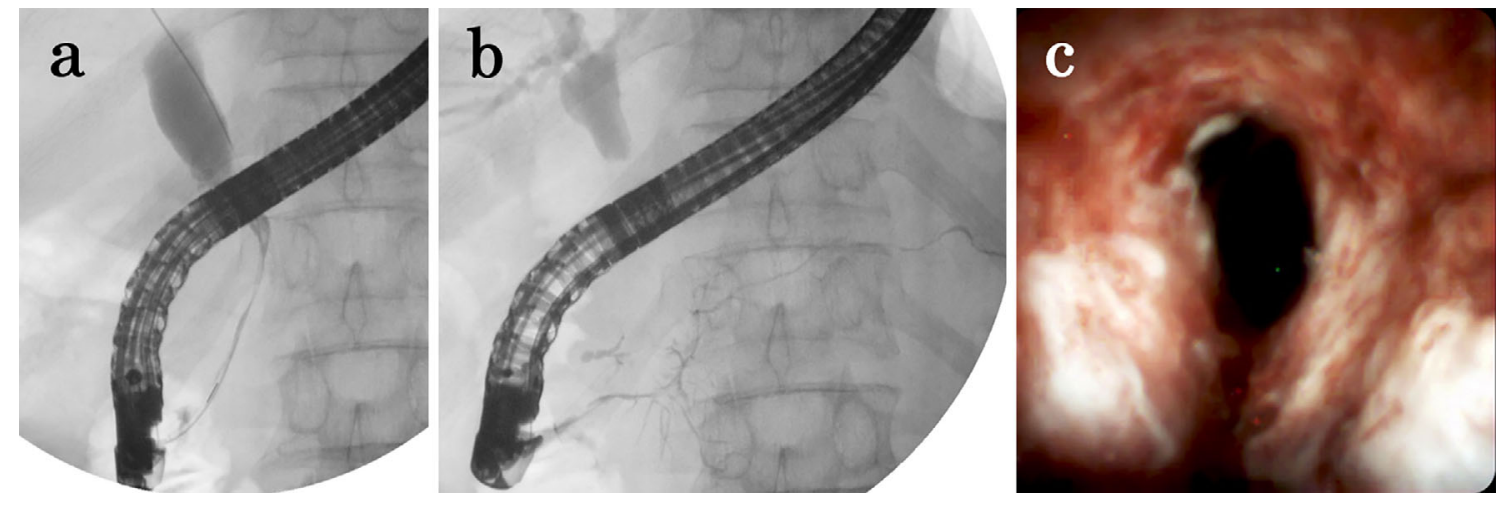

Figure 4. ERCP images demonstrating stenosis of the lower bile duct (a) and narrowing of the main pancreatic duct (b). Peroral cholangioscopy (POCS) revealed fragile, irregular vessels and a nodular elevated surface in the bile duct stricture (c).
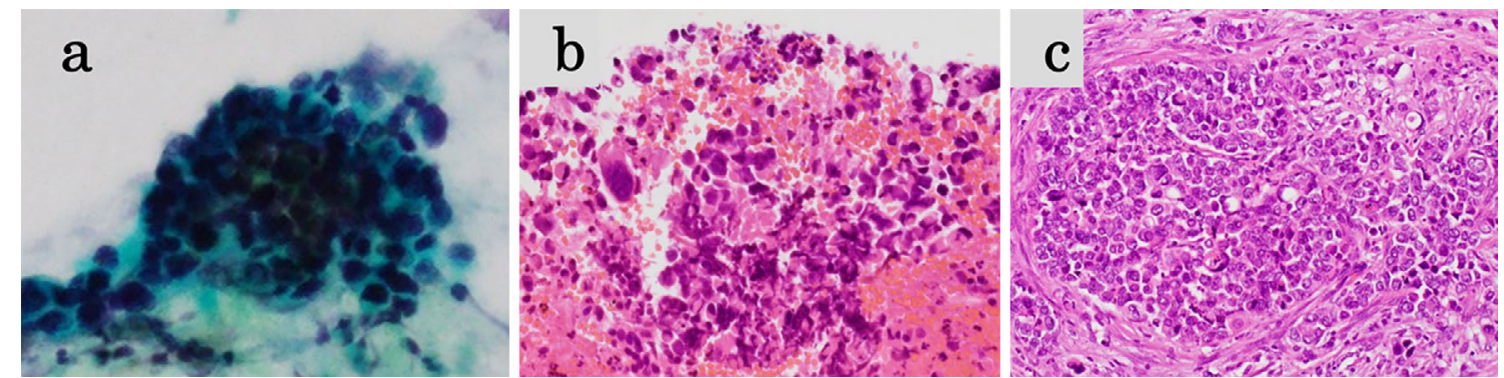

Figure 5. Cytology of the bile juice samples revealing adenocarcinoma cells (a). An EUS-FNAB sample (b) and open-biopsy specimen from cervical lymphadenopathy (c) revealing poorly differentiated adenocarcinoma.

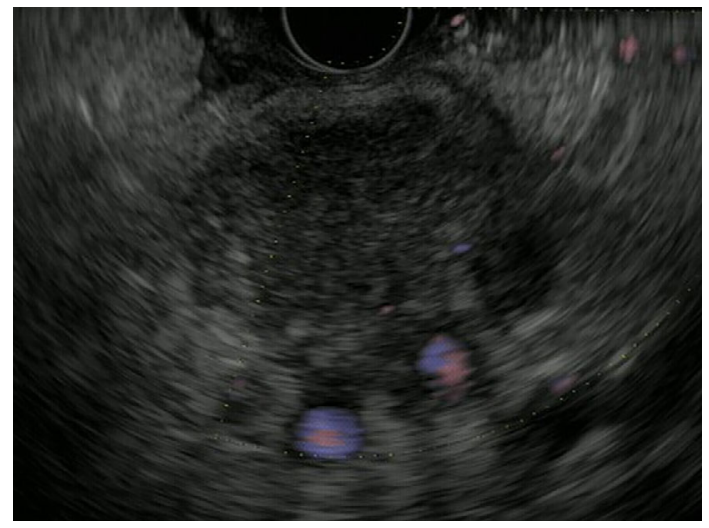

Figure 6. EUS image demonstrating a hypoechoic enlarged pancreas from the head to the body.

(ERCP) demonstrated a stenosis of the lower bile duct (Fig. 4a) and diffuse narrowing of the main pancreatic duct (Fig. 4b). POCS revealed fragile, irregular vessels and a nodular elevated surface in the lower bile duct stricture (Fig. 4c). Suspecting malignancy, we placed an endoscopic nasobiliary drainage (ENBD) catheter for biliary drainage and collection of bile cytology samples, which showed the presence of adenocarcinoma cells (Fig. 5a). Endoscopic ultrasonography (EUS) showed an irregular hypoechoic enlarged pancreas from the head to the body (Fig. 6). Subse- quently, an EUS-FNAB was performed in the pancreatic body, and a histological examination of the samples revealed poorly differentiated adenocarcinoma (Fig. 5b), with findings that were similar to those found in the open-biopsy tissues from cervical lymph nodes (Fig. 5c). No IgG4-positive plasma cells or lymphoplasmacytic sclerosing pancreatitis (LPSP) lesions were observed in any of the biopsy tissues.

The patient was ultimately diagnosed with UICC stage IV PC and was treated with systemic chemotherapy using gemcitabine and nab-paclitaxel following placement of a selfexpandable metallic stent in the lower bile duct stricture.

\section{Discussion}

The differential diagnosis between AIP and PC is crucial because the clinical course, treatment, and prognosis of these two diseases are quite different. Generally, it is easier to differentiate diffuse-type AIP than focal/segmental-type AIP from PC. In this case report, we initially suspected diffuse-type AIP due to the presence of a capsule-like rim at the surface of the sausage-shaped pancreas on CT. We were able to diagnose the malignant lesions more accurately using FDG-PET than CT or MRI. However, recent studies found no significant difference in the SUV of AIP and PC lesions, including both pancreatic and extra-pancreatic lesions $(3,4)$. Regarding the endoscopic results, diffuse irregular narrowing 
on pancreatography and hypoechoic pancreatic enlargement on EUS were also suggestive of AIP. In this case, POCS was more useful than other tools in detecting malignancy as opposed to benign sclerosing cholangitis in AIP $(5,6)$. We were ultimately able to successfully diagnose the patient's PC using FNAB of the pancreatic body after POCS and bile cytology, subsequently clarifying the presence of metastasis by an open biopsy of the cervical lymphadenopathies.

Over the past decade, several diagnostic criteria have been proposed in other countries following the Japan's Pancreas Society (JPS) proposal of the world's first clinical diagnostic criteria for AIP in 2002 (7-9). AIP is usually diagnosed based on a combination of imaging, serological and histological findings, in addition to the involvement of other organs and response to steroids. Since no serum IgG4 elevation was found in our case, the imaging findings described above did not lead to a definitive diagnosis of AIP. According to the International Consensus Diagnostic Criteria (ICDC) for AIP (7), we had the option of performing a diagnostic steroid trial prior to the pathologic confirmation, despite the presence of obstructive jaundice. Bi et al. proposed that obstructive jaundice in AIP could be safely treated with steroids alone, avoiding the use of biliary stents (10). In fact, they placed their patient on steroid therapy for obstructive jaundice in a case of diffuse-type AIP before considering using a biliary stent or having pathology confirmation results. Steroid therapy seems to have delayed appropriate treatments and resulted in the acceleration of the progression of PC in our case, calling attention to the early performance of diagnostic steroid trials without first obtaining pathologic confirmation in patients with AIP.

Several malignant diseases have been reported to exhibit diffusely enlarged pancreas, including pancreatic lymphoma, acinar cell carcinoma, small cell carcinoma, neuroendocrine tumor, and pancreatic metastasis (11-15). AIP has been recognized to lead to development of PC, similar to its occurrence with ordinary chronic pancreatitis (16). PC is, on the other hand, occasionally accompanied by AIP or AIP-like clinical features $(17,18)$. A few recent reports have described paraneoplastic AIP in patients with malignant neoplasms (19). PC can also present an unusual morphology in these conditions. However, the patient in our case did not show elevated serum IgG4 levels or IgG4 positive plasma cells in the biopsy specimens. We believed that his diffusely enlarged pancreas was a result of the replacement of the pancreas with infiltrating tumor cells. Although the histopathologic diagnosis of the pancreas was not confirmed, we based our assumption in the radiological images and EUS results, showing a homogenous pattern without clear margins throughout the enlarged pancreas. In addition, adenocarcinoma cells were confirmed by an FNAB in the body and bile cytologies, indicating that the tumor had diffusely invaded the pancreas from the head to the body.

Diffuse morphology of PC is an unusual manifestation that accounts for approximately $1-5 \%$ of all pancreatic cancers $(20,21)$. The pathologic or radiologic definition of dif- fuse PC (DPC) has not been established because of its low frequency. Previously, Choi et al. summarized cases of DPC (21), reporting that MRCP and/or ERCP in DPC demonstrated normal main pancreatic ducts within the tumors in $5 / 11$ cases $(46 \%)$, segmental irregular narrowing in $2 / 11$ cases $(18 \%)$, and diffuse irregular narrowing in $3 / 11$ cases (27\%), which is compatible with our case findings. This may suggest that the tumor was growing along the duct's subepithelium and invaded the parenchyma diffusely, without destroying the duct's walls. Furthermore, in most DPC cases, contrast-enhanced CT showed "capsule-like structures" that were characterized by an enhanced outer layer in the enlarged pancreas, unlike the capsule-like rim observed in AIP. The hypervascularity was attributed to dense fibrous changes accompanied by fibroblast and lymphocyte infiltration. This finding was not observed in our case, in which the capsule-like rim might have been caused by desmoplastic reactions or inflammatory processes, even though serum amylase elevation and findings of an interrupted pancreatic duct causing pancreatitis were not seen. According to the report, most DPC cases demonstrated direct invasion of neighboring organs, lymphadenopathy, or distant metastasis. As a result of its aggressive characteristics, the mean level of CA 19-9 was elevated approximately 50 times in all cases, whereas it was increased only 2 -fold in our case, despite the presence of systemic lymphadenopathy. Taken together, these results suggest that DPC is diagnosed later or is more aggressive than advanced focal PC.

A number of diagnostic modalities have been developed for differentiating PC from benign lesions, including magnetic resonance elastography, contrast-enhanced harmonic EUS, EUS-elastography, and peroral cholangiopancreatoscopy (22-25). However, these modalities are insufficient at present, and the majority of patients presenting with pancreatic enlargement have benign diseases, while 8-9\% have PC $(26,27)$. Therefore, this report emphasizes the significance of pathological confirmation for pancreatic enlargement, regardless of its focal or diffuse nature, even in the absence of abnormalities on pancreatography, such as stenosis, encasement, or irregular narrowing. In addition, further clinical and pathological evidence supporting the recognition of DPC is needed.

\section{The authors state that they have no Conflict of Interest (COI).}

\section{References}

1. Hardacre JM, Iacobuzio-Donahue CA, Sohn TA, et al. Results of pancreaticoduodenectomy for lymphoplasmacytic sclerosing pancreatitis. Ann Surg 237: 853-859, 2003.

2. Wolfson D, Barkin JS, Chiari ST, et al. Management of pancreatic masses. Pancreas 31: 203-217, 2005.

3. Zhang J, Jia G, Zuo C, Jia N, Wang H. 18F-FDG PET/CT helps differentiate autoimmune pancreatitis from pancreatic cancer. BMC Cancer 17: 695, 2017.

4. Ozaki Y, Oguchi K, Kawa S, et al. Differentiated of autoimmune pancreatitis from suspected pancreatic cancer by fluorine-18 fluro- 
deoxyglucose positron emission tomography. J Gastrienterol 43: 141-151, 2008.

5. Itoi $\mathrm{T}$, Osanai M, Itokawa $\mathrm{F}$, et al. Diagnostic peroral video cholangioscopy is an accurate diagnostic tool for patients with bile duct lesions. Clin Gastroenterol Hepatol 8: 934-938, 2010.

6. Okano N, Igarashi Y, Sasai D, et al. Case of immunoglobulin G4related cholangitis accompanying autoimmune pancreatitis: diagnosis by peroral cholangioscopy and treatment by endoscopic biliary stenting. Dig Endosc 24: 62-66, 2012.

7. Shimosegawa T, Chari ST, Frulloni L, et al. International consensus diagnostic criteria for autoimmune pancreatitis: guidelines of the International Association of Pancreatology. Pancreas 40: 352358, 2011.

8. Okazaki K, Kawa S, Kamisawa T, et al. Clinical diagnostic criteria of autoimmune pancreatitis: revised proposal. J Gastroenterol 44: 626-631, 2006.

9. Chiari ST, Smyrk TC, Levy MJ, et al. Diagnosis of autoimmune pancreatitis: the Mayo Clinic experience. Clin Gastroenterol Hepatorol 4: 1010-1016, 2006.

10. Bi Y, Hart PA, Chiari ST, et al. Obstructive jaundice in autoimmune pancreatitis can be safely treated with corcicosteroids alone without biliary stenting. Pancreatology 16: 391-396, 2016.

11. Magalhães-Costa $P$, Brito $M J$, Pinto-Marques $P$. A diffusely enlarged pancreas: the (un)usual suspect. Rev Esp Enferm Dig 108: 809-811, 2016.

12. Luo Y, Hu G, Li F, et al. Acinar cell carcinoma of the pancreas presenting as diffuse pancreatic enlargement: Two case reports and literature review. Medicine 96: 38(e7904), 2017.

13. Wang RF, Chou YH, Cang CY, et al. Primary small cell carcinoma of the pancreas with an unusual sonographic appearance. J Clin Ultrasound 35: 82-84, 2007.

14. Oscar S, Jesus MM, Ismael DR. Diffuse enlargement of the pancreas: an usual radiologoc presentation of a pancreatic neuroendocrine tumor. Clin Gastroenterol Hepatol 15: e165-e166, 2017.

15. Chou YH, Chiou HJ, Tsay SH, et al. Solitary metastasis from renal cell carcinoma presenting as diffuse pancreatic enlargement. J Clin Ultrasound 30: 499-502, 2002.

16. Ikeura T, Miyoshi H, Okazaki K, et al. Relationship between autoimmune pancreatitis and pancreatic cancer: a single-center experi- ence. Pancreatology 14: 373-379, 2014.

17. Matsubayashi $\mathrm{H}$, Matsunaga $\mathrm{K}$, Ono $\mathrm{H}$, et al. A case of pancreatic carcinoma with suspected autoimmune pancreatitis. Clin J Gastroenterol 2: 59-63, 2009.

18. Inoue $H$, Miyatani $H$, Yoshida $Y$, et al. Pancreatic cancer with autoimmune pancreatitis. Pancreas 33: 208-209, 2006.

19. Lekakis L, Tryfonopoulos D, Efremidis A, et al. A case of paraneoplastic autoimmune pancreatitis: mini-review of paraneoplastic syndromes in breast cancer. Anticancer Res 32: 3311-3314, 2012.

20. Tanaka M. Pancreatic cancer resistry 2007. Suizou 22: e1-e94, 2007 (in Japanese, Abstract in English).

21. Choi YJ, Byun JH, Lee MG, et al. Diffuse pancreatic ductal adenocarcinoma: characteristic imaging features. Eur J Radiol 67: 321-328, 2008.

22. Shi Y, Cang L, Hong Y, et al. The use of magnetic resonance elastography in differentiating autoimmune pancreatitis from pancreatic ductal adenocarcinoma: a preliminary study. Eur J Ragiol 108: 13-20, 2018.

23. Cho MK, Moon SH, Kim MH, et al. Contrast-enhanced endoscopic ultrasound for differentially diagnosing autoimmune pancreatitis and pancreatic cancer. Gut Liver 15: 591-596, 2018.

24. Kanno A, Masamune A, Shimosegawa T. Endoscopic approaches for the diagnosis of autoimmune pancreatitis. Dig Endosc 27: 250258, 2015.

25. Pereira P, Peixoto A, Andrade P, Macedo G. Peroral cholangiopancreatoscopy with the SpyGlass ${ }^{\circledR}$ system: what do we know 10 years later. J Gastrointestin liver Dis 26: 165-170, 2017.

26. Horwhat JD, Gerke H, Jowell PS, et al. Focal or diffuse "fullness" of the pancreas on CT. Usually benign, but EUS plus/minus FNA is warranted to identify malignancy. J Pancreas 10: 37-42, 2009.

27. Ho S, Bonasera RJ, Gress F, et al. A single-center experience of endoscopic ultrasonography for enlarged pancreas on computed tomography. Clin Gastroenterol Hepatol 67: 98-103, 2006.

The Internal Medicine is an Open Access journal distributed under the Creative Commons Attribution-NonCommercial-NoDerivatives 4.0 International License. To view the details of this license, please visit (https://creativecommons.org/licenses/ by-nc-nd/4.0/).

(C) 2019 The Japanese Society of Internal Medicine

Intern Med 58: 2523-2527, 2019 